\title{
Measured Equation of Invariance: A New Concept in Field Computations
}

\author{
Kenneth K. Mei, Fellow, IEEE, Rafael Pous, Member, IEEE, Zhaoqing Chen, \\ Member, IEEE, Yao-Wu Liu, and Mark D. Prouty, Student Member, IEEE
}

\begin{abstract}
Numerical computations of frequency domain field problems or elliptical partial differential equations may be based on differential equations or integral equations. The new concept of field computation presented in this paper is based on the postulate of the existence of linear equations of the discretized nodal values of the fields, different from the conventional equations, but leading to the same solutions. The postulated equations are local and invariant to excitation. It is shown how the equations can be determined by a sequence of "measures." The measured equations are particularly useful at the mesh boundary, where the finite difference methods fail. The measured equations do not assume the physical condition of absorption, so they are also applicable to concave boundaries. Using the measured equations, we can terminate the finite difference mesh very close to the physical boundary and still obtain robust solutions. It will definitely make a great impact on the way we apply finite difference and finite element methods in many problems. Computational results using the measured equations of invariance in two and three dimensions are presented.
\end{abstract}

\section{INTRODUCTION}

$\mathbf{T}$ HE numerical solution of a frequency domain electromagnetic field problem may be approached by an integral equation [1], [2] or a differential equation [3], [4] formulation. The integral equation approach has the advantage of limiting the computational domain on the surface of the object boundary, but it results in a full matrix. The differential equation approach, using a finite difference or finite element method, results in a sparse matrix, but needs a mesh volume much larger than the volume of the object in order to be able to apply an absorbing boundary condition. The sparse matrix of the finite difference method could be a great computational advantage for the differential equation approach if the disadvantage of the excessive mesh coverage does not outweigh the advantage. The ideal case would be to bring the mesh boundary of the finite difference method on to the object boundary. In this paper, we will show that the concept of "measured equation of invariance" (MEI) leads us close to such an ideal situation.

\section{PRIOR InVESTIGATIONS}

It is well known that solutions of linear partial differential

Manuscript received July 24, 1992; revised September 22, 1993. This work was supported by the California State MICRO Program and by MICRO industrial sponsor Hughes Aircraft Company.

The authors are with the Department of Electrical Engineering and Computer Sciences and the Electronics Research Laboratory, University of California, Berkeley, CA 94720.

IEEE Log Number 9215685.

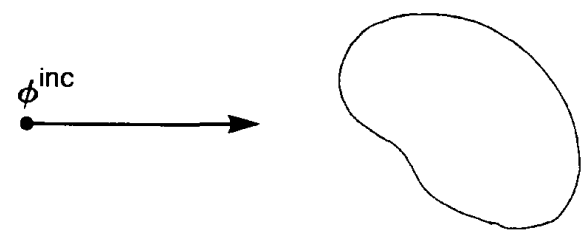

Fig. 1. Schematics of a 2-D scattering.

equations are not unique unless the boundary conditions are specified. The solution of an open domain equation must also include a radiation condition at infinity. Numerically, such a condition is simulated by an absorbing condition at a finite distance from the object boundary. The absorbing boundary condition may be effected by mode matching [4], [5] or by physical considerations [6], [7]. The mode matching techniques are global, and they result in matrices which relate the boundary nodal values to one another. The physical considerations are postulates of absorptions, which are local relations that preserve the sparsity of the difference equations. It has been assumed that if the absorbing conditions were imposed more rigorously, the mesh could be terminated closer to the object surface. Recent investigations by Kriegsman $e t$ al. [8] and Ramahi et al. [9] are representative of such a philosophy. However, the results are mixed. In general, the close to object absorbing conditions are not robust, and they are not applicable to concave surfaces.

\section{THE DifFERENTIAL EQuations}

Let us consider the simple scattering problem of Fig. 1, where the scatterer is a 2-D metal object. The incident field may either be a TE or TM wave. The differential equation to be solved is

$$
\left(\nabla^{2}+k^{2}\right) \phi(\bar{r})=0
$$

where $\phi(\bar{r})$ is the scattered field. The numerical solution of (1) may be found by a set of finite difference equations or finite element equations. Using the typical nodal geometry of Fig. 2, and assuming $h$ to be sufficiently small, the finite difference equation is

$$
\phi_{1}+\phi_{2}-\left(4-k^{2} h^{2}\right) \phi_{0}+\phi_{3}+\phi_{4}=0 \text {. }
$$

We can characterize the finite difference approximation to the differential equation with the following statements. 


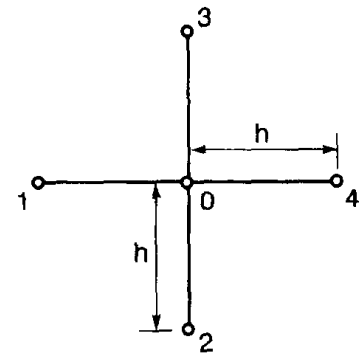

Fig. 2. A typical finite difference mesh point and local numbering.

Each finite difference equation represents a linear equation of a nodal value with its immediate neighbors, and the relation is

1) invariant to the location of the node,

2) invariant to the geometry of the scatterer,

3) invariant to the field of excitation.

The above characterizations also apply to the finite element equations.

\section{THE POStulates}

With reference to the discretized field values of the previous section, Mei advanced the following postulates [10].

For each node, which has $n-1$ immediate neighbors, there exists a linear relation

$$
\sum_{i=0}^{n-1} a_{i} \phi_{i}=0
$$

where $i=0$ is the subscript of the particular node and $i=1,2, \cdots n-1$ are those of the neighbors, which is different from the finite difference and finite element equations at that node. The equations will give the same solution as the discretized differential equation but the equations are

1) location dependent,

2) geometry specific,

3) invariant to the field of excitation.

Postulates 1) and 2) suggest that these equations have to be different from the finite difference and finite element equations. Postulate 3) suggests that the coefficient may be found from $n-1$ linearly independent solutions of the problem. Postulate 2) also suggests that the solutions used to find the coefficients must be specific to the geometry of the scatterer. The above postulates are guidelines upon which we shall depend to converge to a new set of mesh boundary local equations which stays robust near the target boundary. The third postulate seems to be far reaching and incredible, but it is really not that difficult to reason that it is essentially true. All it says is that the linear relation (2) is not the only one at each nodal point. In other words, it is not unique. If it were unique, we should be able to recover the finite different equation from $(n-1)$ solutions obtained from $(n-1)$ different incident fields by substituting them back into (3). In fact, for all scattering problems we know of, such back substitutions do not give us the finite difference equation. Actually, they give us the measured equation of invariance. Our goal, then, is to

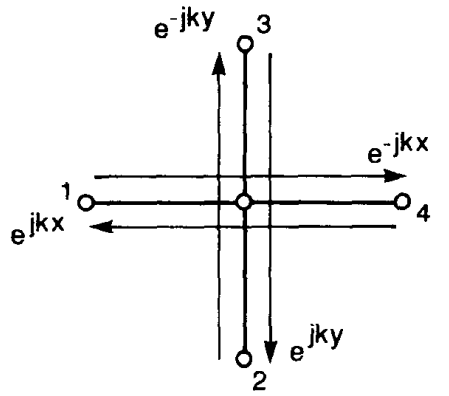

Fig. 3. Orthogonal and opposite plane waves as measuring functions.

find the MEI without solving the problem first. The following sections explain the method.

\section{The Measure}

We shall call a particular solution, which we use to find the coefficients of (3), a "measuring function," and the equation which results from substituting a "measuring function" in (3) a "measure." A minimum of $n-1$ measures are required to get a "measured equation" of $n$ nodes.

A simple example of a measured equation is to use four plane waves as measuring functions on a regular five-point mesh as shown in Fig. 3. The measures are

$$
\begin{aligned}
& a_{1} e^{j k h}+a_{2}+a_{0}+a_{3}+a_{4} e^{-j k h}=0 \\
& a_{1} e^{-j k h}+a_{2}+a_{0}+a_{3}+a_{4} e^{j k h}=0 \\
& a_{1}+a_{2} e^{-j k h}+a_{0}+a_{3} e^{j k h}+a_{4}=0 \\
& a_{1}+a_{2} e^{j k h}+a_{0}+a_{3} e^{-j k h}+a_{4}=0 .
\end{aligned}
$$

Symmetry suggests that $a_{1}=a_{2}=a_{3}=a_{4}$, and since we may choose one coefficient arbitrary, it is convenient to choose $a_{1}=1$. The solution gives

$$
a_{0}=-2-\left(e^{j k h}+e^{-j k h}\right)
$$

The second-order approximation of $a_{0}$ is identical to that of the finite difference equation in (2). This is expected because the above measuring functions are neither location specific nor geometry specific.

A slightly different set of measured equations can be found by using upward plane waves only as measuring functions, such as shown in Fig. 4. The coefficients are now

$$
\left.\begin{array}{l}
a_{0}=-2 \cos k h=\left(k^{2} h^{2}-2\right)+0\left(h^{4}\right) \\
a_{3}=-a_{2}=\frac{\cos \frac{k h}{\sqrt{2}}-\cos k h}{j \sin \frac{k h}{\sqrt{2}}}=\frac{k h}{j 2 \sqrt{2}}+0\left(h^{3}\right) \\
a_{1}=a_{4}=1 .
\end{array}\right\}
$$

In Fig. 4 , we show the residuals of this measured equation when plane waves of arbitrary angular directions are substituted in the equation. It is obvious that Fig. 4 is directional in that it is biased in favor of the upward directed waves. Here, again, we have demonstrated that, as far as the upward directed waves are concerned, the equations of (3) are not unique, and 


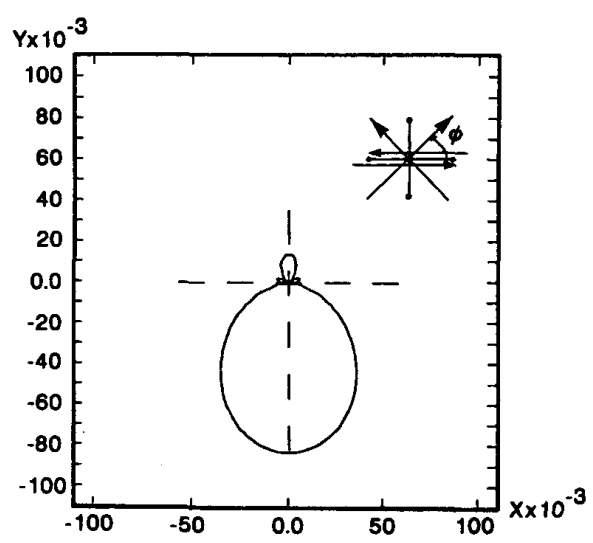

Fig. 4. Four upward plane waves used as measuring functions with $\phi=0^{\circ}$, $45^{\circ}, 135^{\circ}, 180^{\circ}, k h=0.2$, and the residuals of the measured equation for all real angle plane waves.

\section{RESIDUE}

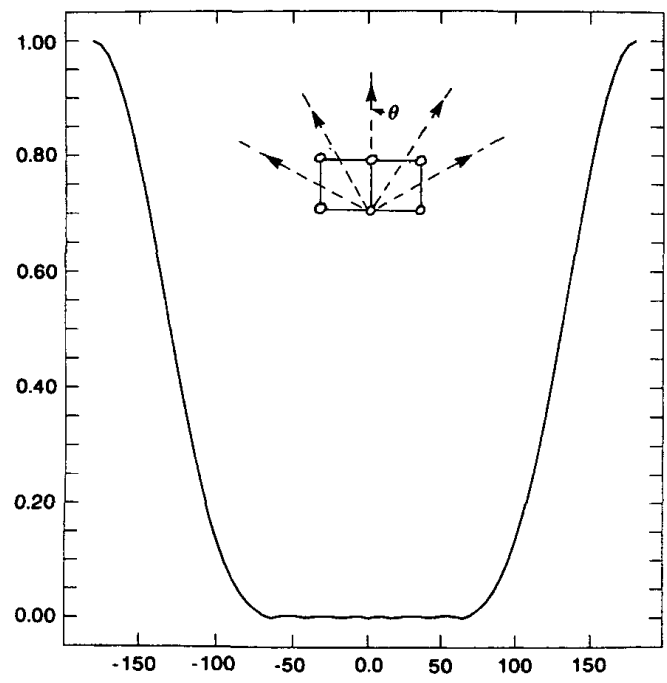

Fig. 5. Residuals of a six-point measured equation using five plane waves as measuring functions with $\theta=0, \pm 35^{\circ}, \pm 65^{\circ}$.

there are equations other than (2) which are capable of locally characterizing the problem and are invariant to incident fields.

Even more directional measured equations can be obtained if we use the six-point mesh as shown in Fig. 5. The residuals of the equation for plane waves are also shown in Fig. 5. Indeed, the measured equation of Fig. 5 is a good radiation boundary condition; however, it is not geometry specific, i.e., the equation is independent of the scatterer geometry. We assert that the near fields are geometry specific, and the equation just discussed cannot be robust near the object boundary.

\section{THE METRONS}

To obtain geometry specific measuring functions, we assume a set of surface current densities $J_{k}\left(\bar{r}^{\prime}\right),(k=1,2, \cdots, n-1)$ on the scatterer surface, called "metrons," and generate the measuring functions from the integral

$$
\phi^{k}(\bar{r})=\int_{c} G\left(\bar{r} \mid \bar{r}^{\prime}\right) J_{k}\left(\bar{r}^{\prime}\right) d c^{\prime}
$$

where $G\left(\bar{r} / \bar{r}^{\prime}\right)$ is the Green's function of (1), and $k=$ $1,2, \cdots, n-1$ for a nodal point having $n-1$ neighbors, $c$ is the contour of the scatterer, $\bar{r}$ is the coordinate location of the node, and $\bar{r}^{\prime}$ is the coordinate location of the metron. It should be emphasized that the metrons are not basis functions. Their sole function is to generate the measured equations. So, there is no requirement for metrons or any linear combination of them to resemble the actual current density. We can use (7) and (3) to generate boundary equations for the finite difference mesh.

If the third postulate is correct, we should be able to terminate a finite difference mesh almost anywhere. It is found that the measured equations mingle well with the conventional finite difference equations, i.e., we may use the finite difference equation at some nodes and MEI at other nodes without adversely affecting the solution. Although we have not tried to mix it with the finite element equations, we have no reason to believe that they will not mix as well. It is frequently advantageous to use the finite difference equation everywhere it is applicable and use MEI where it is necessary, such as at the mesh boundaries. It is also found that the calculations are more stable if there are a few layers of finite difference equations between the object boundary and the mesh boundary. A more stringent integration procedure is needed when the buffer layer is reduced. Normally, robust results can be obtained with just two or three finite difference layers of buffer zone. The extreme case of zero finite difference layer has also been tested and appeared to be robust, although some analytical integration is required because the integrand of (7) becomes singular.

The term "metron" is adapted from the Greek word, which means "measuring instrument." Computationally, a metron current may be considered as an induced surface current due to an unknown incident field. Since the coefficients are independent of incident fields, there is no need for us to find them. What if one uses a metron which cannot be realized by any incident field or if one uses a metron which is the surface current of a resonant cavity mode (which gives zero field outside the cavity)? Of course, one wishes to avoid those cases, and that is why we choose smooth continuous currents as metrons, and we may also choose more metrons than we need and use least square to find the coefficients to overcome any such mishaps.

\section{The Calculations}

We have tested the MEI method on a variety of scatterer geometries in 2-D both for TE and TM cases. Figs. 6 and 7 show the mesh geometry of a square conducting cylinder, which is illuminated by a plane wave, and the calculated surface current densities on the cylinder for the TE and TM cases, respectively. The metrons used in these calculations are

$$
J_{k}(l)=1, \cos \frac{2 k \pi l}{L}, \quad \text { and } \quad \sin \frac{2 k \pi l}{L} \quad(k=1,2)
$$




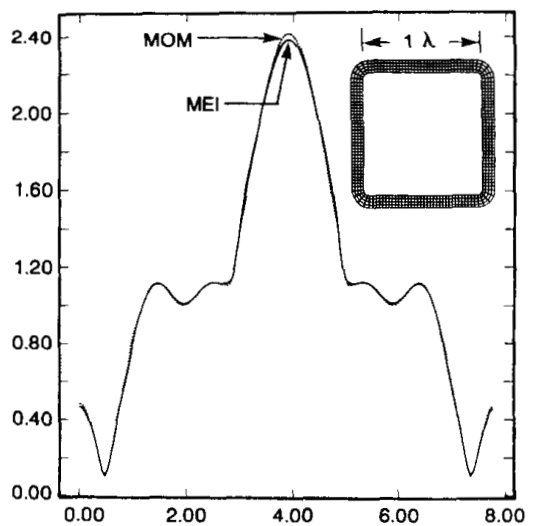

Fig. 6. Mesh geometry and surface current densities on a square conducting cylinder. Comparison of results of a TE case ( $H$ wave) with broad-side incidence.

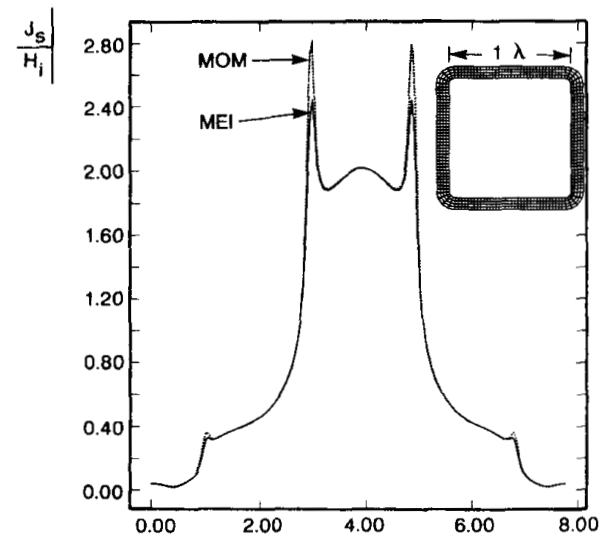

Fig. 7. Mesh geometry and surface current densities on a square conductin cylinder. Comparison of results of a TM case ( $E$ wave) with broad-side incidence.

when the measured functions are generated for a six-point boundary node of Fig. 5 . The letter $l$ denotes the distance measured from the center of the back surface of the cylinder.

It is noticed that the metrons are of slow spatial variation, which cannot possibly represent the results of Fig. 7 which are singular at the corners. The comparisons of the results of the MEI method and MOM are shown, and the agreement is truly remarkable. Fig. 8 shows the mesh geometry of a concave scatterer with a cavity-like indentation. Fig. 9(a) and (b) shows the comparison of the induced surface current densities on the scatterer calculated by the MEI method and by MOM for TM and TE cases, respectively. Again, very robust results are indicated. Metrons similar to (8) are used in these calculations. We notice that the MEI results are slightly asymmetric, while the true results should be perfectly symmetric. That is because two of the metrons we have used are not symmetric. Since the measured equations of invariance are not exact due to discretization, one would expect some numerical errors to creep in. Of course, had we used only symmetrical metrons, we would also get perfectly symmetrical results.

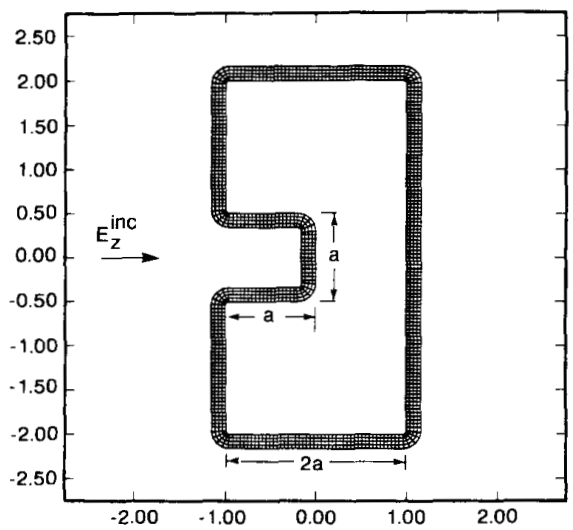

Fig. 8. Geometry and mesh of a scatterer with a cavity-like indentation.

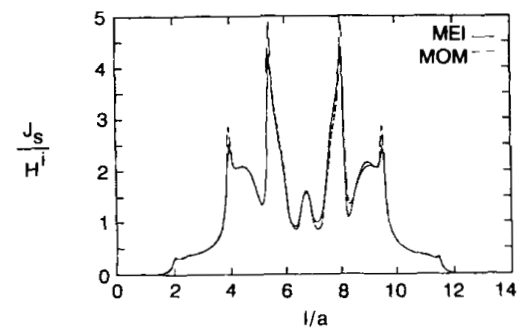

(a)

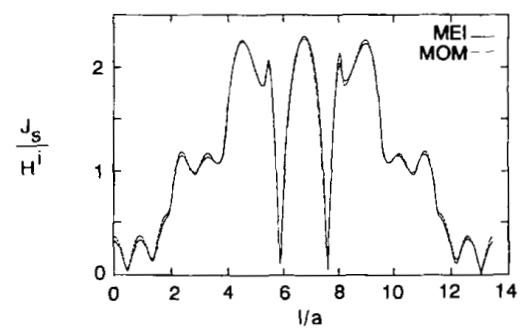

(b)

Fig. 9. The surface current densities on the scatterer of Fig. 8 for $\lambda=1.8 a$ and angle of incidence $\varphi=0^{\circ}$ as calculated by MEI method and MOM. (a) TM-wave incidence, (b) TE-wave incidence.

As a test of the MEI method without any finite difference buffer, Fig. 10 shows the geometry and mesh of an elliptical scatterer, and Figs. 11 and 12 show the calculated results with zero and one finite difference buffer and their comparisons with those of MOM. Although a few layers of finite difference buffer zone do not seem to add much computational overhead in the 2-D case, they do make a great difference in 3-D cases. As more experiences are gained in the MEI computations, it is expected that the importance of the buffer zone should diminish.

While the above results indicate that the MEI method and MOM have comparable accuracy, the need for computer time and memory is vastly different. For storage, the MOM requires $N \times N$ words of memory, yet the MEI method needs only 


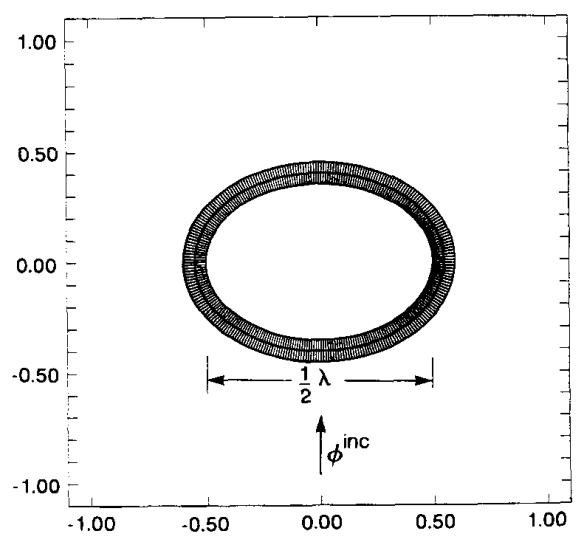

Fig. 10. Mesh and geometry of an elliptical cylinder with one finite difference buffer layer.

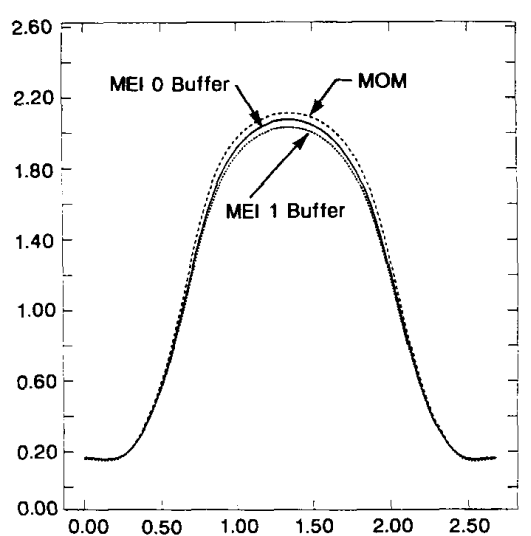

Fig. 11. Calculated surface current densities on the conducting elliptical cylinder using MEI with 0 and 1 finite difference buffer layers, and their comparisons with that of the MOM (TM wave).

$n^{2} \times N$, where $m=n-1$ is the number of buffer layers. For $n=4$ and $N=100$, for example, MOM requires six times more memory than the MEI method, 12 times of $N=200$, etc. The time advantage of the MEI is equally dramatic. Fig. 13 shows some typical time for the cases we have tested, indicating that MOM time is on the order of $N^{2.5}$ and MEI about $N^{1.9}$. For an honest comparison, the $N$ in both MOM and MEI are the number of nodal points on the surface of the target, i.e., the same $N$ for MOM and $\mathrm{MEI}$ are used even though there are more unknowns for MEI than for MOM. We realize that Fig. 13 is only the plot for relatively small $N$. When $N \rightarrow \infty$, the MOM slope must approach 3 and the MEI slope must approach 2. Most of the computation time for the MEI method is spent on the calculation of the measured equation of invariance at the mesh boundary. Such calculations depend on the generation of the measuring functions of (7), which for each nodal point requires the same amount of calculations for the method of moments. It is noted that the calculations for more than one metron do not

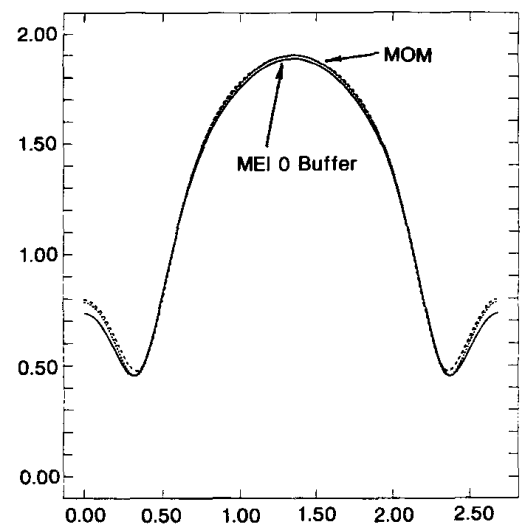

Fig. 12. Calculated surface current densities on the conducting elliptical cylinder using MEI with 0 and 1 finite difference buffer layers, and their comparisons with that of the MOM (TE wave).

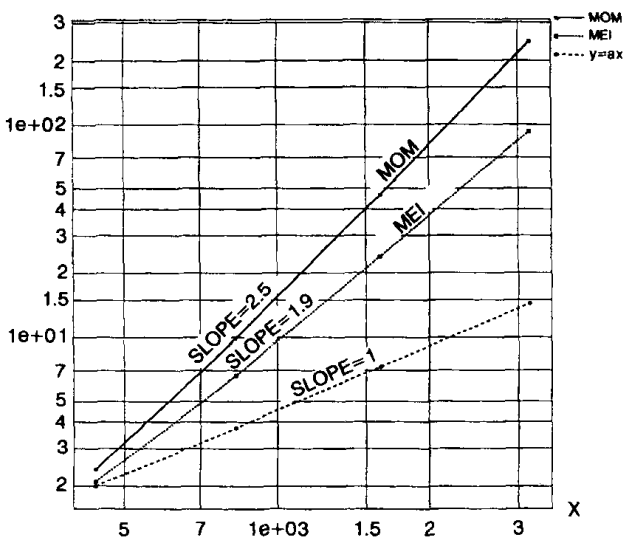

Fig. 13. Time versus $N$ plots of MOM and MEI.

add much overhead to the work for only one metron because different metrons only result in different constant multipliers of the Green's functions at each summation step. It is not required to recalculate the Green's function. It is not difficult to see that $2 / 3$ of the calculations are redundant. Therefore, for a six-point boundary MEI, we require twice the amount of time needed for MOM to fill the matrix. We believe that there is much room for advancement in the calculation of the measuring functions of (7). In the MOM research activities, we have generally neglected the research in integration because in the MOM, the computation is dominated by the matrix inversion so there has been little incentive to speed up the calculation of the matrix.

In the extreme case of $m=0$ and 1, the actual CPU seconds for results of Figs. 11 and 12 are those shown in Table I.

The advantage of the MEI method will be even more dramatic when applied to 3-D problems.

\section{THE COROLLARIES}

In the above calculations, we have confirmed the veracity of the postulates; in particular, it is demonstrated in Figs. 8 and 
TABLE I

Actual CPU Seconds for Results of FIGS. 11 AND 12.

\begin{tabular}{lcc}
\hline Methods & TM CPU seconds & TE CPU seconds \\
\hline MOM & 34 & 58 \\
MEI $(m=1)$ & 9.4 & 9.4 \\
MEI $(m=0)$ & 7.6 & 7.6 \\
\hline
\end{tabular}

The DEC $5000 / 200 \mathrm{cx}$ workstation was used for the above calculations.

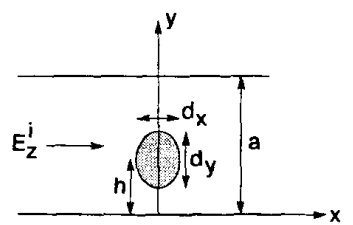

(a)

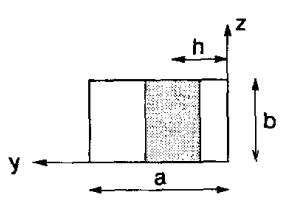

(b)

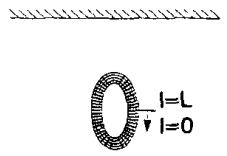

(c)

Fig. 14. The scattering configuration of an elliptical cylinder obstacle in a rectangular waveguide. (a) Top view, (b) end view, (c) mesh.

9 that the measured equation of invariance is definitely not another absorbing boundary condition since there exist both incoming and outgoing waves at those boundaries. It further suggests that the MEI can be applied in closed regions such as waveguides and cavities, without extending the mesh to the device boundaries. In this section, we shall present a few corollaries of the MEI method.

\section{A. Mesh Flexibility}

One of the inconveniences of the finite difference method is that for the five-point mesh (seven points for 3-D), the mesh must cross at right angles, which reduces its flexibility to conform to object boundaries. The MEI method provides an easy way out, where mesh flexibility is needed.

\section{B. Utility of the Green's Functions}

By changing the Green's function in (7), which generates the measuring functions, we have immediately changed the scattering environment of the problem with no other overhead. As an example, we calculated the surface current densities on

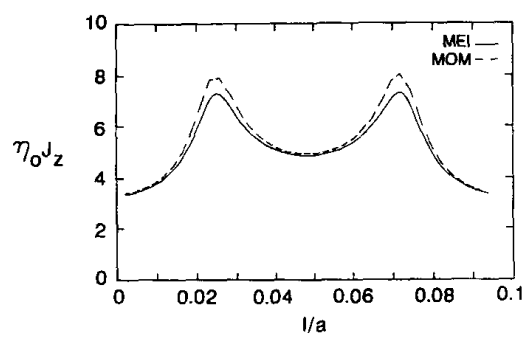

(a)

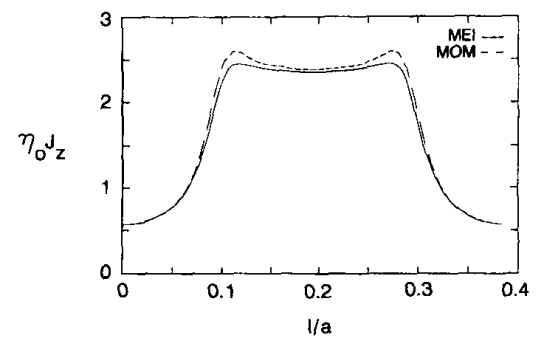

(b)

Fig. 15. Surface current density around the perimeter of the elliptical post for $\lambda=1.5 a$ for two values of the diameter $2 d_{x}=d_{y}=d$ compared to the MOM. (a) $d=0.04 a$, (b) $d=0.16 a$.

an elliptical post in a rectangular waveguide illuminated by a $\mathrm{TE}_{01}$ incident wave. The scattering configuration is shown in Fig. 14. The novelty of this calculation is that the only difference between it and the free space scattering calculation is the Green's function. It is noticed that the mesh in Fig. 14 conforms only to the scatterer, but not to the waveguide. The conventional finite difference or finite element methods would require a mesh to conform to both the object and device boundaries, which requires a demanding programming effort. The comparison of the MEI results for the surface current densities with the MOM results are shown in Fig. 15, and the comparisons of $S_{11}$ of MEI calculations to those of the variational results are shown in Fig. 16.

\section{Mesh Umbilical}

An interesting case is posed by the scattering of waves by multiple bodies when we use the MEI method. In that case, the natural thing to do is to use metron pairs in finding the boundary MEI. However, after the boundary equations are found, the equations between one object boundary and its mesh boundary appear to be self-consistent. That would lead to an illogical conclusion that the current on one body can be obtained independently of that on the other. The truth of the matter is that in any scattering configuration, we are not allowed to use disconnected mesh groups. For a multibody configuration, the mesh groups must be connected by mesh umbilicals. The charge densities on a pair of coupled microstrip lines have been calculated as an illustration. In Fig. 17, the strips and mesh configuration are shown, and Fig. 18 shows the charge distribution on the grounded middle strip. The errors are the differences between the results of a calculation with a full mesh and one with only umbilical connections. The comparisons of the resulting capacitances 


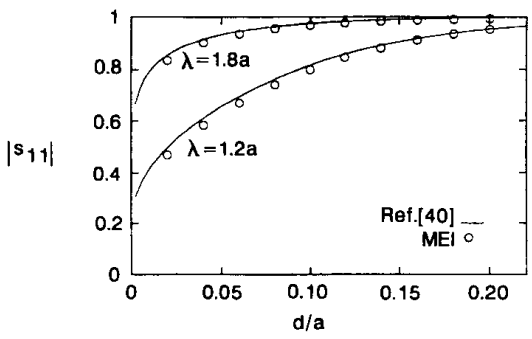

(a)

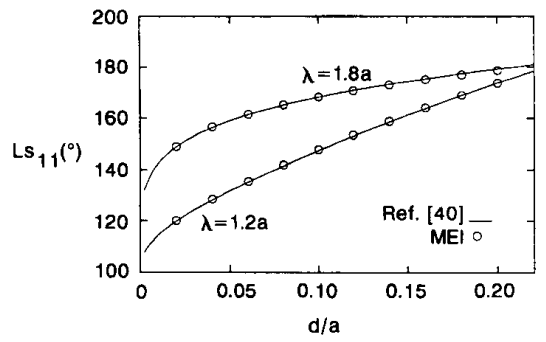

(b)

Fig. 16. Reflection coefficient for an elliptical post of diameter $2 d_{x}=d_{y}=d$, placed at the center of the waveguide $(h=a / 2)$ for two different values of $\lambda$, compared to the variational result in [14]. (a) Magnitude, (b) phase.

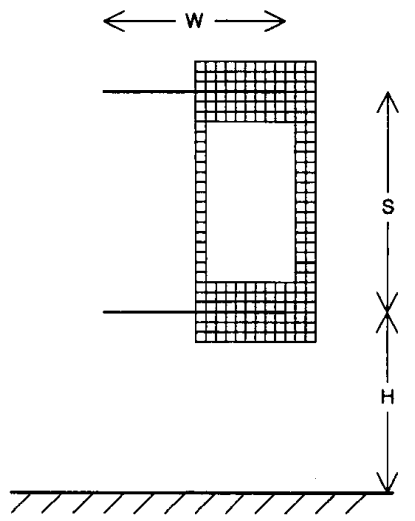

Fig. 17. Two mesh groups connected by mesh umbilicals for a pair of parallel strips above a ground plane.

with those of the spectral domain are shown in the table with Fig. 18, and they are very close.

\section{THE EXTENSION TO 3-D}

The extension of the MEI method of 3-D is conceptually immediate, even though the effort in programming is greatly increased. In this paper, we shall only give a few representative results and leave the details of computations to separate papers. Figs. 19 and 20 are the computational configurations and results of a tape dipole and a sheet scatterer, respectively. Fig. 18 is significant in that it shows that the MEI method is also robust in solving antenna problems, where the source is a "delta generator" [11]. It is noticed that this computation also includes the transverse profile of the current densities
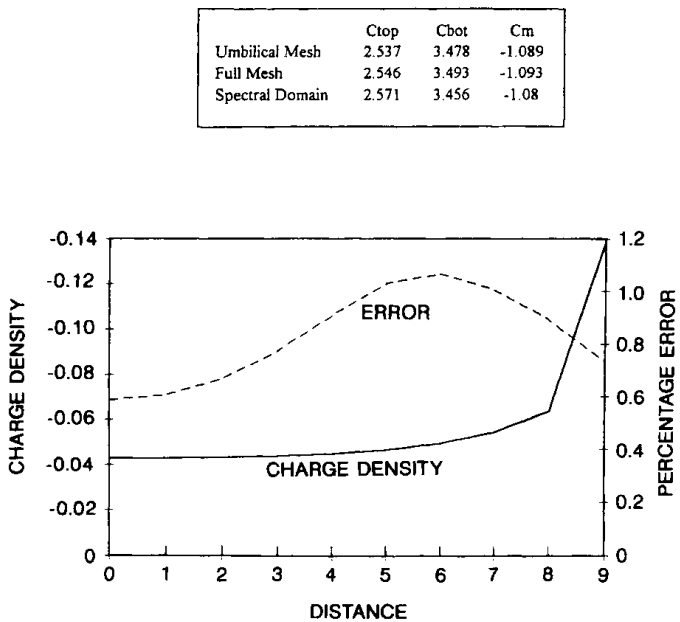

Fig. 18. Capacitances, charge densities (on middle conductor), and errors of charge densities for the pair of parallel strips of Fig. $16 .(H=18, S=22$, $w=18$.)
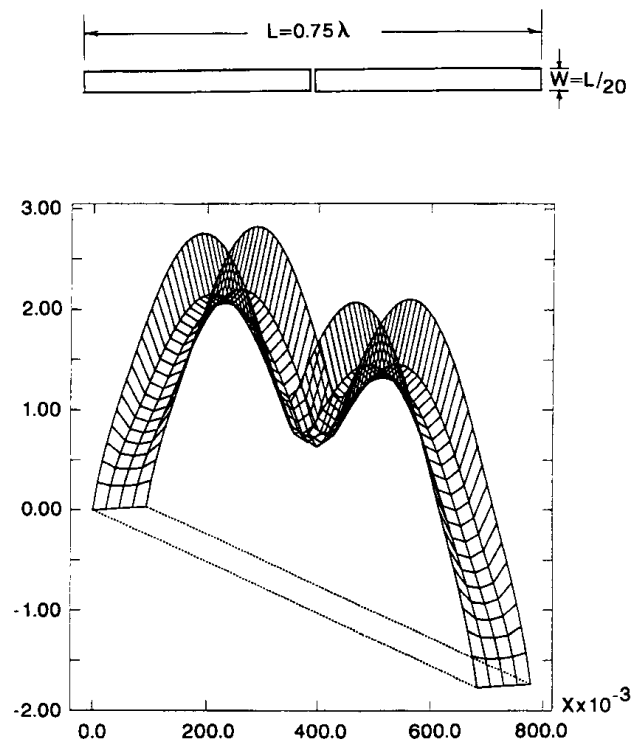

Fig. 19. A flat strip dipole antenna and surface current densities as it is driven by a delta generator.

of the flat dipole, which is frequently "assumed" in the moment calculations [12]. The results of the radar cross section calculated from the current density of Fig. 20 of the card scatterer also compares very favorably with that of the MOM result given by Tsai [13].

\section{$X$. THE IMPACT}

The impact of the concept of measured equations of invariance on electromagnetic field computation is quite evident. It compels us to reconsider the traditional methods of field computation, such as finite difference, finite element, and the method of moments. Indeed, it should have significant influences on all field computations, where elliptical partial 

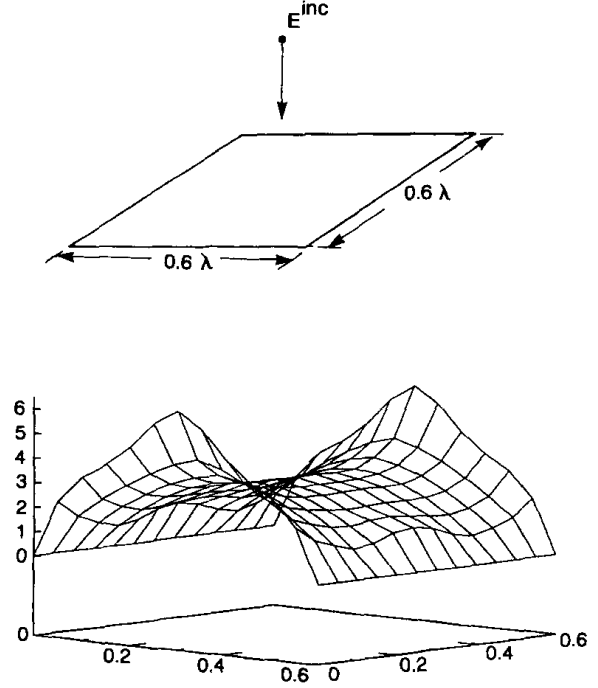

Fig. 20. Scattering configuration and the amplitudes of induced current densities on a square metal plate.

differential equations are involved. The advantages in computational time and memory of the MEI method over the traditional method such as those demonstrated in this paper are already quite significant, but we feel it is still a beginning. For instance, in the MOM approach, the greatest consumer of CPU time is the calculation of the inversion of a large full matrix; in the MEI method, the most demanding part is the calculation of the matrix elements. It should be noted that each measured equation of invariance is an independent calculation, so the method will greatly benefit from the future MPC (massively parallel computation) systems. We should expect even greater impact in the reduction of time and memory as more research is done on this new method.

\section{REFERENCES}

[1] K. K. Mei and J. G. Van Bladel, "Scattering by perfectly conducting rectangular cylinders," IEEE Trans. Antenn. Propagat., vol. AP-1 I, pp. 185-192dd, Mar., 1963.

[2] R. F. Harrington, Field Computation by Moment Method. New York, Macmillan 1968.

[3] K. K. Mei, "Unimoment method of solving antennas and scattering problems," IEEE Trans. Antenn. Propagat., vol. AP-22, pp. 760-766, Nov. 1974.

[4] M. A. Morgan, "Finite element computation of scattering by inhomogeneous penetrable bodies of revolutions," IEEE Trans. Antenn. Propagat., vol. AP-27, pp. 202-214, Mar. 1979.

[5] M. A. Morgan and P. W. Barber, "Finite-element boundary-integral formulation for electromagnetic scattering," Int. J. Wave Motion, pp. 91-103, Jan. 1984

[6] A. Bayliss and E. Turkel, "Radiation boundary conditions for wave-like equations," Commun. Pure Appl. Math., vol. 33, pp. 707-725, 1980.

[7] B. Engquist and A. Majda, "Absorbing boundary conditions for the numerical simulation of waves," Math. Comput., vol. 31, pp. 629-651, July 1977.

[8] G. A Kriegsman, A. Taflove, and K R Umashankar, "A new formulation of electromagnetic wave scattering using a surface radiation boundary condition approach," IEEE Trans. Antenn. Propagat., vol. AP-35, pp. 152-161, Feb. 1987.

[9] O. M. Ramahi, A. Khebir, and R. Mittra, "Numerically derived ab. sorbing boundary condition for the solution of open region scattering problem," IEEE Trans. Antenn. Propagat., vol. 39, pp. 350-354, Mar. 1991.
[10] K. K. Mei, presented at the weekly research meeting of the EM Group of the EECS Dep., Univ. California, Berkeley.

[11] R. W. P. King, Theory of Linear Antennas. Cambridge, MA, Harvard Univ. Press, 1956.

[12] P. B. Katehi and N. G. Alexopoulos, "Frequency-dependent characteristics of microstrip discontinuities in millimeter-wave integrated circuits," IEEE Trans. Microwave Theory Tech., vol. MTT-33, pp. 1029-1035, Oct. 1985.

[13] L. L. Tsai, "Radar cross section of a simple target: A three-dimensional conducting rectangular box," IEEE Trans. Antenn. Propagat., vol. AP00, pp. 882-884, Nov. 1977.

[14] N. Marcuvitz, Waveguide Handbook. New York, McGraw-Hill, 1951.

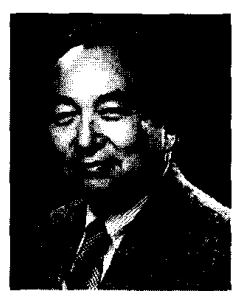

Kenneth K. Mei (S'61-M'63-SM'76-F'79) received the B.S.E.E., M.S., and Ph.D. degrees in electrical engineering from the University of Wisconsin, Madison, in 1959, 1960, and 1962 respectively.

He joined the faculty of the Department of Electrical Engineering and Computer Sciences of the University of California, Berkeley, in 1962 where he is now a Professor. His main areas of interest are antennas, scattering, electromagnetic theories, and numerical methods of solving field problems. He began research on computational methods in electromagnetics in 1960, and his interest in computations includes the method of moments, finite element/difference methods, hybrid methods, time domain methods, and the measured equation of invariance.

Dr. Mei received the Best Paper Award in 1967 and Honorable Mention from the Best Paper Award in 1967 and 1975, respectively, from the IEEE Antennas and Propagation Society. He is a member of the URSI/USNC and was a memory of the AP-S Ad Com and an Associate Editor of its TRANSACTIONS.

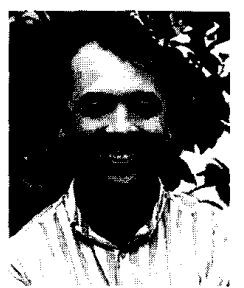

Rafael Pous (S'89-M'93) was born in Barcelona, Spain, in 1964. He received the Ingeniers degree in telecommunication engineering and the Licenciado degree in computer science from the Polytechnic University of Catalonia, Barcelona, in 1988, and University of Catalonia, Barcelona, in 1988, and
the Ph.D. degree in electrical engineering from the University of California, Berkeley, in 1992. He was awarded the Fulbright Scholarship and the Schlumberger Fellowship during his studies.

In 1993 he joined the Telecommunication Engineering Faculty of the Polytechnic University of Catalonia, where is is currently an Assistant Professor. His research interests are in the areas of computational electromagnetics and applied superconductivity.

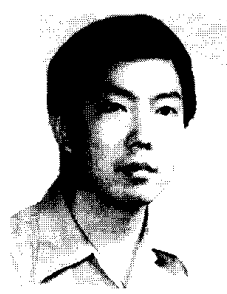

Zhaoqing Chen (M'92) was born in Nanchang, China on January 18,1956 . He received the B.S. degree from Jiangxi University, Nanchang, China, in 1982 and the M.Eng. and Ph.D. degrees from Tinghua University, Beijing, China, in 1985 and 1989, respectively.

He was with the ERL of the University of California, Berkeley, in 1991 and 1992 . He is now with the Department of Electronic Engineering, Tsinghua University, Beijing. China, where he is an Associate Professor. His research interests are in modeling microwave and high-speed integrated circuits components, computational electromagnetics, and the development of MIC/MMIC CAD programs.

Dr. Chen received (jointly with coauthors) the Outstanding Paper Award in 1986 from the Chinese Institute of Electronics (CIE). He is a coauthor of the book, Computer-Aided Design of Microwave Circuits (in Chinese, Tsinghua University Press, 1988) 
I

8 